\title{
RANDOM NONLINEAR EVOLUTION INCLUSIONS IN REFLEXIVE BANACH SPACES
}

\author{
EVGENIOS P. AVGERINOS AND NIKOLAOS S. PAPAGEORGIOU ${ }^{1,2}$
}

(Communicated by William D. Sudderth)

\begin{abstract}
In this paper we present two existence results for a large class of random, nonlinear, multivalued evolution equations defined in a reflexive, separable Banach space and involving an $m$-dissipative operator. Applications to random multivalued parabolic p.d.e.'s are presented. Our work unifies and extends earlier results of Kampé de Feriet, Gopalsamy and Bharucha-Reid, Becus and Itoh.
\end{abstract}

1. Introduction. Many problems in physics, engineering, biology and social sciences lead to mathematical equations. In these equations, the coefficients and the other parameters have their origin in experimental data and represent some kind of average value. Therefore in many instances due to wide variations of the data or even due to our own ignorance, it is appropriate to abandon the deterministic model in favor of a stochastic one. This is very nicely exemplified in the books of Bharucha-Reid [4] and Soong [21].

In this paper we present two existence results for a class of random nonlinear multivalued evolution equations defined in a reflexive, separable Banach space. This class of evolution equations models linear and several nonlinear partial differential equations of parabolic type. So our work unifies and extends earlier works on random parabolic partial differential equations. In particular, Kampé de Feriet $[12,13]$ was the first to study the random heat equation. The randomness entered in the problem through the initial value data. Later Gopalsamy and Bharucha-Reid [10] and Becus [2] introduced also randomness in the boundary value and source terms. Finally Itoh [11], allowed randomness to appear also in the operator, which instead of the Laplacian, was a general random, single valued, everywhere defined continuous, accretive operator. Our results cover all the above-mentioned works.

Let $(\Omega, \Sigma)$ be a measurable space and $X$ a separable Banach space. Throughout this work we will be using the following notations: $P_{f(c)}(X)=\{A \subseteq X$ : nonempty, closed, (convex) $\}$ and $P_{k c}(X)=\{A \subseteq X$ : nonempty, compact, convex $\}$. A multifunction $F: \Omega \rightarrow P_{f}(X)$ is said to be measurable if for every $x \in X$, $\omega \rightarrow d(x, F(\omega))=\inf \{\|x-z\|: z \in F(\omega)\}$ is measurable. By $S_{F}^{1}$ we will denote the set of integrable selectors of $F(\cdot)$ i.e. $S_{F}^{1}=\left\{f \in L^{1}(X): f(\omega) \in F(\omega) \mu\right.$-a.e. $\}$.

Received by the editors August 18, 1987.

1980 Mathematics Subject Classification (1985 Revision). Primary 60H20.

Key words and phrases. $m$-dissipative, integral solution, measurable multifunction, measurable selector, nonlinear semigroup of contractions.

${ }^{1}$ Research supported by N.S.F. Grant D.M.S. 8602313

${ }^{2}$ Work done while on leave at the University of Thessaloniki, School of Technology, Mathematics Division, Thessaloniki 54006, Greece. 
This set may be empty. We will say that $F(\cdot)$ is integrably bounded if and only if $\omega \rightarrow|F(\omega)|=\sup \{\|z\|: z \in F(\omega)\}$ belongs in $L^{1}$. It is easy to check that in this case $S_{F}^{1} \neq \varnothing$.

Let $J: X \rightarrow 2^{X^{*}}-\{\varnothing\}$ be the duality map, that is

$$
J(x)=\left\{x^{*} \in X^{*}:\left(x^{*}, x\right)=\|x\|^{2}=\left\|x^{*}\right\|^{2}\right\} .
$$

Then for $(x, y) \in X \times X$ we define

$$
(x, y)_{+}=\sup \left\{\left(y^{*}, x\right): y^{*} \in J(y)\right\} .
$$

This is called a semi-inner product on $X$. Consider the initial value problem $\dot{x}(t) \in A x(t)+f(t), x(0)=x_{0}$, where $A: X \rightarrow 2^{X}$ is $m$-dissipative, $f \in L^{1}(X)$ and $x_{0} \in \overline{D(A)}$. A function $x: T=[0, b] \rightarrow X$ is an integral solution of the above equation, if $x(\cdot)$ is continuous, $x(0)=x_{0}$ and

$$
\|x(t)-x\|^{2} \leq\|x(s)-x\|^{2}+2 \int_{s}^{t}(f(r)+y, x(r)-x)_{+} d r
$$

for each $(x, y) \in \operatorname{Gr} A$ and $0 \leq s \leq t \leq b$. If $A$ is linear, then the family of integral solutions coincides with that of mild solutions. A strong solution is an integral inclusion. The converse is not true without additional hypotheses on $X, A, f$ and $x_{0}$. It is true, if $X=R^{n}$ or if $X$ is a Hilbert space and $-A=\partial \varphi, \varphi(\cdot)=$ proper, convex, closed function on $X$. Further results in this direction can be found in Brezis [5], Barbu [1] and Schechter [20]. Recall that given an $m$-dissipative operator $A: X \rightarrow 2^{X}$, it generates a nonlinear semigroup $\{S(t), t \geq 0\}$ of contractions on $\overline{D(A)}$, through the Crandall-Liggett exponential formula [1]

$$
S(t) x=\lim _{n \rightarrow \infty}\left(I-\frac{t}{n} A\right) x^{-n} .
$$

Finally if $Y, Z$ are Hausdorff topological spaces, a multifunction $G: Y \rightarrow 2^{Z}$ $\{\varnothing\}$ is said to be closed if and only if $\operatorname{Gr} G=\{(y, z) \in Y \times Z: z \in G(y)\}$ is closed in $Y \times Z$.

2. The theorem. Let $(\Omega, \Sigma, \mu)$ be a complete probability space, $T=[0, b]$ a closed, bounded interval in $R_{+}$and $X$ a reflexive, separable Banach space. With $X_{w}$ we will denote $X$ with the weak topology. The random multivalued, nonlinear evolution equation that we will consider is

$$
\begin{gathered}
\dot{x}(\omega, t) \in A(\omega) x(\omega, t)+F(\omega, t, x(\omega, t)), \\
x(\omega, 0)=x_{0}(\omega) .
\end{gathered}
$$

In the above equation, for every $\omega \in \Omega, A(\omega): X \rightarrow 2^{X}$ is an $m$-dissipative operator, while $F(\cdot, \cdot, \cdot)$ is a random multivalued perturbation. By a random integral solution of $(*)$ we understand a stochastic process $x: \Omega \times T \rightarrow X$ with continuous realizations s.t. for every $\omega \in \Omega, x(\omega, \cdot)$ is an integral solution of $x(\omega, t) \in A(\omega) x(\omega, t)+f(\omega)(t)$, where $f(\omega)(\cdot) \in S_{F(\omega, \cdot, x(\omega, \cdot))}^{1}$.

ThEOREM 2.1. Assume (1) for every $\omega \in \Omega, A(\omega): X \rightarrow 2^{X}$ is an m-dissipative operator that generates a semigroup $S(\omega)(t)$ which is compact for $t>0$ and $\omega \rightarrow \operatorname{Gr} A(\omega)$ is measurable from $\Omega$ into $P_{f}(X \times X)$. 
Let $F: \Omega \times T \times X \rightarrow P_{f_{c}}(X)$ be such that

(2) $(\omega, t, x) \rightarrow F(\omega, t, x)$ is measurable,

(3) for every $(\omega, t) \in \Omega \times T, x \rightarrow F(\omega, t, x)$ is closed in $X \times X_{w}$,

(4) $|F(\omega, t, x)| \leq \alpha(\omega, t)$ a.e., where $\alpha(\cdot, \cdot)$ is jointly measurable and for all $\omega \in \Omega$, $\alpha(\omega, \cdot) \in L^{1}$, and

(5) $x_{0}: \Omega \rightarrow X$ is measurable and for all $\omega \in \Omega, x_{0}(\omega) \in \overline{D(A(\omega))}$.

Then (*) admits a random integral solution.

Proof. For each $\omega \in \Omega$ and $g \in L^{1}(X)$, consider the following evolution equation:

$(*)_{\omega}$

$$
x(t) \in A(\omega) x(t)+g(t), \quad x(0)=x_{0}(\omega) .
$$

From Benilan [3], we know that $(*)_{\omega}$ has a unique integral solution. Let $H: \Omega \times$ $L^{1}(X) \rightarrow C(T, X)$ be the map that to each $(\omega, g) \in \Omega \times L^{1}(X)$ assigns the unique integral solution. We will examine the properties of the map $H(\cdot, \cdot)$. Fix $g \in L^{1}(X)$. From Lemma 2.1, p. 124 of Barbu [1], we know that $H(\omega, g)(t)=\lim _{n \rightarrow \infty} x_{n}(\omega, t)$ uniformly on $T$, where $x_{n}(\omega, t)$ is the unique solution of the approximate evolution equation.

$$
\dot{x}_{n}(\omega, t)=A_{n}(\omega) x_{n}(\omega, t)+g(t), \quad x_{n}(\omega, 0)=x_{0}(\omega) .
$$

Recall that $A_{n}(\omega) x=n\left(F_{n}(\omega) x-x\right)$, where $F_{n}(\omega) x=\left(I-n^{-1} A(\omega)\right)^{-1} x$ and $x \in R\left(I-n^{-1} A(\omega)\right)$, and so combining hypothesis (1) with Theorem 1 of Menou [15], we get that $\omega \rightarrow A_{n}(\omega)$ is measurable. So $\omega \rightarrow x_{n}(\omega, t), n \geq 1$, are measurable $\Rightarrow \omega \rightarrow H(\omega, g)$ is measurable.

Now fix $\omega \in \Omega$. From inequality (2.4), p. 124 of Barbu [1], we get that $g \rightarrow H(\omega, g)$ is continuous from $L^{1}(X)$ into $C(T, X)$. So $(\omega, g) \rightarrow H(\omega, g)$ is a Carathéodory function, hence jointly measurable.

Next let $R: \Omega \times L^{1}(X) \rightarrow P_{f c}\left(L^{1}(X)\right)$ be the multifunction defined by $R(\omega, g)=$ $S_{F(\omega,,, H(\omega, g)(\cdot))}^{1}$. We claim that $\operatorname{Gr} R \in \Sigma \times B\left(L^{1}(X)\right)$ (i.e. $R(\cdot, \cdot)$ is graph measurable). To this end, rewrite $R(\cdot, \cdot)$ as

$$
R(\omega, g)=\left\{f \in L^{1}(X): d\left(f, S_{F(\omega, \cdot, H(\omega, g)(\cdot))}^{1}\right)=0\right\} .
$$

From the definition of the distance function we have

$$
\begin{aligned}
d\left(f, S_{F(\omega,,, H(\omega, g)(\cdot))}^{1}\right) & =\inf \left\{\|f-h\|_{1}: h \in S_{F(\omega, \cdot, H(\omega, g)(\cdot))}^{1}\right\} \\
& =\inf \left\{\int_{0}^{b}\|f(t)-h(t)\| d t: h \in S_{F(\omega, \cdot, H(\omega, g)(\cdot))}^{1}\right\} \\
& =\int_{0}^{b} \inf \{\|f(t)-z\|: z \in F(\omega, t, H(\omega, g)(t))\} d t \\
& =\int_{0}^{b} d(f(t), F(\omega, t, H(\omega, g)(t))) d t .
\end{aligned}
$$

Observe that $H(\omega, g)(t)=e_{t}(H(\omega, g))$, where $e_{t}(\cdot)$ is the evaluation map at $t \in T$, which we know is jointly continuous. Also we saw earlier that $H(\omega, g)$ is jointly measurable. Thus $(\omega, t, g) \rightarrow H(\omega, g)(t)=e_{t}(H(\omega, g))=p(\omega, t, g)$ is measurable and so using hypothesis (2) we deduce that for every $x \in X,(\omega, t, g) \rightarrow$ $d(x, F(\omega, t, H(\omega, g)(t)))$ is measurable. Since the distance function is continuous 
in $x$, we conclude that $(\omega, t, g) \rightarrow d(f(t), F(\omega, t, H(\omega, g)(t)))$ is measurable and so through Fubini's theorem we get that $(\omega, g) \rightarrow d\left(f, S_{F(\omega, \cdot, H(\omega, g)(\cdot))}^{1}\right)$ is measurable and once again the continuity in $f$ allows us to say that $(\omega, g, f) \rightarrow$ $d\left(f, S_{F(\omega, \cdot, H(\omega, g)(\cdot))}^{1}\right)=k(\omega, g, f)$ is jointly measurable. Then we have:

$$
\begin{aligned}
\operatorname{Gr} R & =\left\{(\omega, g, f) \in \Omega \times L^{1}(X): k(\omega, g, f)=0\right\} \\
& \in \Sigma \times B\left(L^{1}(X) \times L^{1}(X)\right)=\Sigma \times B\left(L^{1}(X)\right) \times B\left(L^{1}(X)\right) .
\end{aligned}
$$

Observe that for every $\omega \in \Omega, R(\omega, \cdot): W(\omega) \rightarrow W(\omega)$, where $W(\omega)_{=} S_{B(\omega, \cdot)}^{1}$ with $B(\omega, t)=\{x \in X:\|x\| \leq \alpha(\omega, t)\}$. Note that since $X$ is reflexive, $W(\omega)$ is $w$-compact in $L^{1}(X)$ (Dunford-Pettis theorem). Then since $S(\omega)(t)$ is compact for $t>0$, Theorem 2 of Vrabie [22] tells us that $\overline{H(\omega, W(\omega))}$ is compact in $C(T, X)$. So if $g_{n} \stackrel{w}{\rightarrow} g$ in $W(\omega)$, we may say that $H\left(\omega, g_{n}\right) \rightarrow \hat{x}$ in $C(T, X)$ and using once more inequality (2.4), p. 124 in Barbu [1], we get that $\hat{x}=H(\omega, g)$. Then from hypothesis (3) and Theorem 4.2 of [17] we get that $w-\overline{\lim } R\left(\omega, g_{n}\right) \subseteq R(\omega, g) \Rightarrow R(\omega, \cdot)$ is u.s.c. on $W(\omega)$. Hence applying Kakutani-Ky Fan fixed point theorem we get that $L(\omega)=\{f \in W(\omega): f \in R(\omega, f)\} \neq \varnothing$ for all $\omega \in \Omega$. Note that $\operatorname{Gr} L=$ $\operatorname{proj}_{\Omega \times L^{1}(X)}[(\Omega \times D) \cap \operatorname{Gr} R]$ where $D=\left\{\left(f_{1}, f_{2}\right) \in L^{1}(X) \times L^{1}(X): f_{1}=f_{2}\right\}$. From Theorem 39.IV.1 of Kuratowski [14] (see also Dellacherie [9], Theorem A, p. 345-the Arsenin-Novikov theorem) and since Gr $R \in \Sigma \times B\left(L^{1}(X)\right) \times B\left(L^{1}(X)\right)$, we get that $\operatorname{Gr} L \in \Sigma \times B\left(L^{1}(X)\right)$. Apply the Aumann selection theorem (see SaintBeuve [19], Theorem 3) to get $\hat{h}: \Omega \rightarrow L^{1}(X)$ measurable s.t. $\hat{h}(\omega) \in L(\omega)$ for all $\omega \in \Omega$. Let $\hat{x}(\omega, t)=H(\omega, \hat{h}(\omega))(t)$. Clearly $\hat{x}(\cdot, \cdot)$ is the desired random integral solution of $(*)$. Q.E.D.

We can relax the compactness hypothesis on $S(t), t>0$, on the expense of introducing it on the perturbation $F(\cdot, \cdot, \cdot)$. Furthermore we assume that $X$ is a separable Hilbert space.

THEOREM 2.2. Assume (1) for every $\omega \in \Omega,-A(\omega): X \rightarrow 2^{X}$ is maximal monotone with int $D(A(\omega)) \neq \varnothing$ and $\omega \rightarrow \operatorname{Gr} A(\omega)$ is measurable from $\Omega$ into $P_{f}(X \times X)$.

Let $F: \Omega \times T \times X \rightarrow P_{f c}(X)$ be such that

(2) $(\omega, t, x) \rightarrow F(\omega, t, x)$ is measurable,

(3) for every $(\omega, t) \in \Omega \times T, x \rightarrow F(\omega, t, x)$ is closed in $X \times X_{w}$,

(4) $F(\omega, t, x) \subseteq G(\omega, t)$, where $G: \Omega \times T \rightarrow P_{k c}(X)$ is measurable and integrably bounded in $t$, and

(5) $x_{0}: \Omega \rightarrow X$ is measurable and for all $\omega \in \Omega, x_{0}(\omega) \in \overline{D(A(\omega))}$. Then $(*)$ admits a random integral solution.

ProOF. As in the proof of Theorem 2.1 we consider the multifunction $R(\omega, g)$ which is graph measurable. Also for every $\omega \in \Omega, R(\omega, \cdot): W(\omega) \rightarrow W(\omega)$ and in order to apply the Kakutani-Ky Fan fixed point theorem, we need to show that it is upper semicontinuous on $W(\omega)=S_{G(\omega, \cdot)}^{1}$ with the weak topology. So let $g_{n} \stackrel{w}{\rightarrow} g$ in $W(\omega)$. Using the theorem of Castaing [8], we get that since $G(\cdot, \cdot)$ is compact valued, weak convergence of sequences coincides with convergence in the weak norm $|g|_{w}=\max \left\{\left\|\int_{t}^{t^{\prime}} g(s) d s\right\|: t, t^{\prime} \in T\right\}$. Hence $g_{n} \stackrel{|\cdot|_{w}}{\rightarrow} g$ and so through the theorem in $\S 4$ of Schechter [20], we get that $H\left(\omega, g_{n}\right) \rightarrow H(\omega, g)$ in $C(T, X)$. The rest of the proof goes as in Theorem 2.1. Q.E.D. 
3. Applications. (1) Let $(\Omega, \Sigma, \mu)$ be a complete probability space and $W$ an open domain in $R^{n}(n>2)$ with smooth boundary $\partial W=\Gamma$. Let $r>(n-2) / n$ and consider the following random, nonlinear multivalued initial-boundary value problem of parabolic type on $\Omega \times T \times W$ :

$(* *)_{1}$

$$
\begin{gathered}
\frac{\partial x(\omega, t, z)}{\partial t} \in \lambda(\omega) \Delta x(\omega, t, z)|x(\omega, t, z)|^{r-1}+F(\omega, t, z, x(\omega, t, z)) \\
x(\omega, t, z)=0 \quad \text { on } \Omega \times T \times \Gamma \\
x(\omega, 0, z)=x_{0}(\omega, z) \quad \text { on } \Omega \times\{0\} \times W .
\end{gathered}
$$

Here $\lambda: \Omega \rightarrow R$ is measurable, $F: \Omega \times T \times R^{n} \times R \rightarrow P_{f c}(R)$ is a closed multifunction s.t. $|F(\omega, t, z, x)| \leq \alpha(\omega, t, z)$ with $\alpha$ measurable, $\alpha(\omega, \cdot, \cdot) \in L^{2}(T \times W)$ and $(\omega, t, x) \rightarrow S_{F(\omega, t, \cdot, x(\cdot))}^{1}$ measurable on $\Omega \times T \times L^{2}(W)$.

Set $X=L^{2}(W)$ and $A(\omega) x=\lambda(\omega) \Delta x|x|^{r-1}$ with domain $D(A(\omega))=\{x \in$ $L^{2}(W): x,|x|^{r-1} \in H_{0}^{1}(W)$ and $\left.\Delta x|x|^{r-1} \in L^{2}(W)\right\}$. Then from Benilan [3] we know that for every $\omega \in \Omega, A(\omega)$ is $m$-dissipative while using Corollary 5.1, p. 214 of Pavel [18] (see also Brezis [7]), we can conclude that the semigroup that it generates is compact for $t>0$. Furthermore it is clear that $\omega \rightarrow \operatorname{Gr} A(\omega)$ is measurable from $\Omega$ into $P_{f}(X \times X)$. Finally let $\widehat{F}: \Omega \times T \times X \rightarrow P_{f c}(X)$ be defined by $F(\omega, t, x)=S_{F(\omega, t, \cdot, x(\cdot))}^{1}$. From the hypotheses on $F(\cdot, \cdot, \cdot, \cdot)$ we can check easily that $\widehat{F}$ satisfies assumptions (2), (3), and (4) of Theorem 2.1. Setting $\hat{x}_{0}(\omega)=x_{0}(\omega, \cdot) \in L^{2}(W)$ we can rewrite $(* *)_{1}$ as follows

$$
\begin{gathered}
\dot{x}(\omega, t) \in A(\omega) x(\omega, t)+\widehat{F}(\omega, t, x(\omega, t)), \\
x(\omega, 0)=\hat{x}_{0}(\omega) .
\end{gathered}
$$

Applying Theorem 2.1, we deduce that $(* *)_{1}^{\prime}$ (and so $\left.(* *)_{1}\right)$ has a random integral solution, which is a strong solution since $A(\omega)$ is of subdifferential type (see Example 2).

(2). Let $W$ be an open domain in $R^{n}$ with smooth boundary and consider the following problem on $\Omega \times T \times W$ :

$$
\begin{gathered}
\frac{\partial x(\omega, t, z)}{t} \in \Delta \beta(\omega, x(\omega, t, z))+f(\omega, t, z, x(\omega, t, z)), \\
0 \in \beta(\omega, x(\omega, t, z)) \quad \text { on } \Omega \times T \times \Gamma, \\
x(\omega, 0, z)=x_{0}(\omega, z) \quad \text { on } \Omega \times\{0\} \times W .
\end{gathered}
$$

Here $\beta(\omega)=\partial h(\omega, \cdot)=$ the subdifferential of a Carathéodory integrand $h: \Omega \times$ $R \rightarrow R$. Also $f: \Omega \times T \times R^{n} \times R \rightarrow R$ is measurable in $(\omega, t, z)$, continuous in $x$ and $f(\omega, t, z, x) \in K(\omega, t)$, where $K: \Omega \times T \rightarrow P_{f c}(R)$ is measurable and for all $\omega \in \Omega,|K(\omega, \cdot)| \in L_{+}^{2}$. Let $\varphi: \Omega \times H^{-1}(W) \rightarrow R \cup\{+\infty\}$ be defined by

$$
\varphi(\omega, x)=\left\{\begin{array}{l}
\int_{W} h(\omega, x(z)) d z, \quad x \in L^{1}(W), h(\omega, x(\cdot)) \in L^{1}(W), \\
+\infty \text { otherwise. }
\end{array}\right.
$$

Recall that $\Delta$ is the canonical isomorphism between $H_{0}^{1}(W)$ and $H^{-1}(W)=$ $\left[H_{0}^{1}(W)\right]^{*}$ and apply Proposition 2.10, p. 67, of Barbu $[1]$ to rewrite $(* *)_{2}$ as follows:

$$
\begin{gathered}
\dot{x}(\omega, t) \in-\partial \varphi(\omega, x(\omega, t))+\widehat{F}(\omega, t, x(\omega, t)), \\
x(\omega, 0)=x_{0}(\omega)(\cdot) \in H^{-1}(W),
\end{gathered}
$$


where $\widehat{F}$ is the Nemitsky operator associated with $f$. Then applying Theorem 2.2, we deduce that $(* *)_{2}^{\prime}$ being of subdifferential type has a random strong solution (which is in fact unique since $\widehat{F}$ is single valued)

$$
x: \Omega \times T \rightarrow H^{-1}(W) \text { s.t. } \sqrt{t}(\partial x(\omega, t) / \partial t) \in L^{2}\left(T, H^{-1}(W)\right) .
$$

Clearly then $x(\omega, t, z)=x(\omega, t)(z)$ is the desired distributional random solution of $(* *)_{2}$.

Note that if $h(\omega, x)=\lambda(\omega) x|x|^{r-1}$ we recover example (1). That is why the solution in that example is in fact strong. Also depending on the form of $h(\omega, x)$ we can have various interesting problems in mathematical physics (free boundary problems, flow through homogeneous porous medium etc.)

(3). Let $\Omega, W$ be as before. Consider the problem

$$
\begin{gathered}
\frac{\partial x(\omega, t, z)}{t}=\Delta x(\omega, t, z)+f(\omega, t, z, x(\omega, t, z)), \\
x(\omega, t, z)=0 \quad \text { on } \Omega \times T \times \Gamma, \\
x(\omega, 0, z)=x_{0}(\omega, z) \quad \text { on } \Omega \times\{0\} \times W .
\end{gathered}
$$

Here $f: \Omega \times T \times R^{n} \times R \rightarrow R$ is measurable in $(\omega, t, z)$, continuous in $x$ and $|f(\omega, t, z, x)| \leq \alpha(\omega, t, z)$ with $\alpha(\cdot, \cdot, \cdot)$ measurable, $\alpha(\omega, \cdot, \cdot) \in L^{2}(T \times W)$. It is well known that if $A=\Delta$ with $D(A)=H_{0}^{1}(W) \cap H^{2}(W)$, then $A(\cdot)$ is dissipative on $L^{2}(W)$. Furthermore from the existence theory of partial differential equations we know that $R(I-\Delta)=L^{2}(W)$. So $A$ is an $m$-dissipative operator. In addition from Brezis [6] we know that $-A=\partial \varphi$ where $\varphi(x)=\frac{1}{2} \int_{W}|\nabla x(z)|^{2} d z$ if $x(\cdot) \in H_{0}^{1}(\Omega)$, and $+\infty$ otherwise. Then system $(* *)_{3}$ takes the form:

$$
\begin{gathered}
\dot{x}(\omega, t)=A x(\omega, t)+F(\omega, t, x(\omega, t)), \\
x_{0}(\omega, 0)=\hat{x}_{0}(\omega),
\end{gathered}
$$

where $F(\omega, t, x)$ is the Nemitsky operator corresponding to $f$ and $\hat{x}_{0}(\omega)(\cdot)=$ $x_{0}(\omega, \cdot) \in L^{2}(W)$. Recalling that $A$ generates a linear contractions semigroup $S(t): L^{2}(W) \rightarrow L^{2}(W)$ which is compact for $t>0$, we conclude using Theorem 2.1, that $(* *)_{3}$ has a random strong solution (it is strong because $\left.A=\partial \varphi\right) x: \Omega \times T \rightarrow$ $L^{2}(W)$ s.t. $\sqrt{t}(\partial x(\omega, t) / \partial t) \in L^{2}\left(T, L^{2}(W)\right)$ and $x(\omega)(\cdot) \in L^{2}\left(T, H_{0}^{1}(W)\right)$. Then $x(\omega, t)(\cdot)$ is the desired random distributional solution of $(* *)_{3}$. This example illustrates that our work produces as special cases the existence results of Kampé de Feriet [12, 13], Gopalsamy and Bharucha-Reid [10] and Becus [2].

(4). If $X$ is a Hilbert space, $K: \Omega \rightarrow P_{k c}(X)$ is measurable and $\delta_{K(\omega)}(\cdot)$ is the indicator function of $K(\omega)$ i.e. $\delta_{K(\omega)}(x)=0$ if $x \in K(\omega),+\infty$ otherwise, we consider the following random multivalued equation

$$
\begin{gathered}
\dot{x}(\omega, t) \in \partial \delta_{K(\omega)}(x(\omega, t))+f(\omega, t, x(\omega, t)), \\
x(\omega, 0)=x_{0}(\omega) \in X,
\end{gathered}
$$

where $f: \Omega \times T \times X \rightarrow X$ is measurable in $(\omega, t)$, continuous in $x$ and $\|f(\omega, t, x)\| \leq$ $\alpha(\omega, t)$ a.e. with $\alpha(\cdot, \cdot)$ measurable and $\alpha(\omega, \cdot) \in L^{1}$. Recall that $\partial \delta_{K(\omega)}=N_{K(\omega)}=$ cone of normals to $K(\omega)$. Hence in equation $(* *)_{4}$ we recognize the "sweeping process problem" considered by Moreau [16], that has applications in theoretical mechanics. If $A(\omega)=\partial \delta_{K(\omega)}(\cdot)$ then $A(\omega)$ is maximal monotone and by Corollary 5.1, 
p. 214 of Pavel [18] generates a compact nonlinear semigroup. So Theorem 2.2 guarantees the existence of a random strong solution for $(* *)_{4}$.

ACKNOWLEDGMENT. The authors are grateful to the referee for his (her) corrections and remarks.

\section{REFERENCES}

1. V. Barbu, Nonlinear semigroups and differential equations in Banach spaces, Noordhoff, Leyden, 1976.

2. G. Becus, Random generalized solutions to the heat equation, J. Math. Anal. Appl. 90 (1977), 93-102.

3. P. Benilan, Equations d'evolution dans un espace de Banach quelconque et applications, Thése, Université de Paris XI, Orsay, 1972.

4. A. Bharucha-Reid, Random integral equations, Academic Press, New York, 1972.

5. H. Brezis, Operateurs maximaux monotones et semigroupes de contractions dans les espaces de Hilbert, Math. Studies 5, North-Holland, Amsterdam, 1973.

6. __ Monotonicity methods in Hilbert spaces and some applications to nonlinear partial differential equations, Contributions to Nonlinear Functional Analysis (E. Zarantonello, ed.), Academic Press, New York, 1971.

7. _ New results concerning monotone operators and nonlinear semigroups, R.I.M.S. Kyoto Univ., No. 258, pp. 2-27.

8. C. Castaing, Un resultat de compacité lié á la propriété des ensembles Dunford-Pettis dans $L_{F}^{1}(\Omega)$, Seminaire d'Analyse Convexe, Montpellier 1979, Exp. No. 17.

9. C. Dellacherie, Ensembles analytiques: Théorèmes de separation et applications, Seminaire de Probabilités IX, Université de Strasbourg, Lecture Notes in Math., vol. 465, Springer-Verlag, Berlin and New York, 1975.

10. K. Gopalsamy and A. Bharucha-Reid, On a class of parabolic differential equations driven by stochastic point processes, J. Appl. Prob. 12 (1975), 98-106.

11. S. Itoh, Random differential equations associated with accretive operators, J. Differential Equations 31 (1979), 139-154.

12. J. Kampé de Feriet, Intégrales aleatoires de l'equation de la chaleur dans une barre infinie, C. R. Acad. Sci. Paris 240 (1955), 710-712.

13. _ Random solutions of partial differential equations, Proc. 3rd Berkeley Sympos. Math. Stat. and Prob., Vol. III, Univ. of California Press, Berkeley, 1956, pp. 199-208.

14. K. Kuratowski, Topology I, Academic Press, New York, 1966.

15. S. Menou, Famille mesurable d'operateurs maximaux monotones, C. R. Acad. Sci. Paris 290 (1980), 711-714.

16. J.-J. Moreau, Evolution problem associated with a moving convex set in a Hilbert space, J. Differential Equations 26 (1977), 347-374.

17. N. S. Papageorgiou, Convergence theorems for Banach space valued integrable multifunctions, Internat. J. Math. Math. Sci. 10 (1987), 433-442.

18. N. Pavel, Differential equations, flow invariance and applications, Research Notes in Math., vol. 113, Pitman, London, 1984.

19. M.-F. Saint-Beuve, On the extension of Von Neumann-Aumann's theorem, J. Funct. Anal. 17 (1974), 112-129.

20. E. Schechter, Perturbations of regularizing maximal monotone operators, Israel J. Math 43 (1982), 49-61; correction, Israel J. Math. 47 (1984), 236-240.

21. T. Soong, Random differential equations in science and engineering, Academic Press, New York, 1973.

22. I. Vrabie, Nonlinear evolution equations: Existence via compactness (H. Brezis, M. Crandall and F. Kappel, eds.), Pitman, New York, 1986, pp. 242-248.

Department of Mathematics, University of Thessaloniki, School of TeChNOLOGY, THESSALONIKI 54006 GREECE 95616

Department of Mathematics, University of California, Davis, California 